\title{
Hemithyroidectomy increases the risk of disease recurrence in patients with ipsilateral multifocal papillary thyroid carcinoma
}

\author{
XIAOLONG LI $^{1 *}$, CUI ZHAO ${ }^{1 *}$, DANDAN HU $^{1}$, YANG YU $^{1}, \mathrm{JIN} \mathrm{GAO}^{2}$, WENCHUAN ZHAO $^{1}$ and MING GAO ${ }^{1}$ \\ ${ }^{1}$ Department of Head and Neck Tumor, Tianjin Medical University Cancer Institute and Hospital, Tianjin 300060, P.R. China; \\ ${ }^{2}$ School of Medicine and Dentistry, James Cook University, Douglas, Queensland 4878, Australia
}

Received October 31, 2012; Accepted January 31, 2013

DOI: $10.3892 / \mathrm{ol} .2013 .1202$

\begin{abstract}
Papillary thyroid carcinoma (PTC) is often clinically multifocal. In this study, the clinicopathological characteristics of a total of 347 PTC patients treated between 2006 and 2007 were investigated in order to assess the risk factors for tumor recurrence in patients with multifocal PTC. Of all the PTC cases reviewed, 35 (10\%) were categorized as multifocal PTC. Patients with multifocal PTC were significantly more likely to have extrathyroidal extension, lymph node metastases and disease recurrence $(\mathrm{P}<0.05)$. Hemithyroidectomy resulted in a significantly higher incidence of tumor recurrence in patients with ipsilateral multifocal PTC compared with unifocal PTC patients $(\mathrm{P}<0.01)$. In conclusion, hemithyroidectomy was associated with tumor recurrence in patients with ipsilateral multifocal PTC but not those with unifocal PTC. Hemithyroidectomy should only be carried out after careful deliberation when involving patients with ipsilateral multifocal PTC.
\end{abstract}

\section{Introduction}

Papillary and follicular carcinoma are defined as differentiated thyroid carcinomas derived from thyroid follicular epithelial cells and account for $>90 \%$ of all thyroid cancers (1-3). Papillary thyroid carcinoma (PTC) is significantly more common than follicular thyroid carcinoma and is characterized by a high rate of lymph node metastases (4-9). The incidence of multifocal PTC has been increasing rapidly $(10,11)$.

There has been significant debate as to whether multifocal PTC patients should undergo aggressive surgical therapy.

Correspondence to: Dr Ming Gao or Dr Xiaolong Li, Department of Head and Neck Tumor, Tianjin Medical University Cancer Institute and Hospital, Tianjin 300060, P.R. China

E-mail: law.li@tom.com

E-mail: leonlee_tianjin@hotmail.com

*Contributed equally

Key words: papillary thyroid cancer, multifocal, recurrence, hemithyroidectomy
Certain studies have reported that PTC tumors should receive bilateral thyroidectomy and radioablation of the remaining tissue according to the finding that papillary cancer foci may have independent origins $(10,12,13)$. Others have suggested that this treatment is too aggressive since multicentricity is not considered to be a prognostic determinant for the categorization of tumors as low or high risk (14). Furthermore, there is no consistent opinion on the treatment of central neck compartment lymph node metastases. Although the significance of therapeutic central neck dissection (CND) is undisputed, there is no consensus on the role of elective CND in clinically node-negative patients with multifocal PTC (15). To investigate this issue, a retrospective study of 347 cases of PTC, including 35 of multifocal PTC, was performed to determine the risk factors for multifocal PTC relative to known prognostic variables.

\section{Materials and methods}

Patients. A single institution retrospective review of patients with PTC was performed. The clinical data of a total of 347 patients treated for PTC between January 2006 and December 2007 at Tianjin Medical University Cancer Institute and Hospital (Tianjin, China) were investigated. None of the patients had a history of prior head and neck irradiation. All the patients underwent planned total thyroidectomy or hemithryoidectomy and CND or lateral neck dissection. Data on gender, age, histological variants, extrathyroidal extension, lymph node metastases and recurrence were available for all 347 patients. The present study was approved by the Tianjin Medical University Cancer Institute and Hospital Institutional Review Board. Written informed consent was obtained from the patients.

Treatment and follow-up. Prior to surgery, every patient underwent a CT scan of the head and neck and an ultrasonographic evaluation of the thyroid and cervical lymph node. Fine-needle aspiration (FNA) of the primary tumor or intraoperative examination of frozen tissue sections was performed routinely to obtain the diagnosis of the primary disease. Frozen sections (2-3) were regularly analyzed depending on the tumor size. The surgical procedure was determined according to the results of the preoperative and intraoperative evaluations. Total thyroidectomy was performed on patients with any of the 
Table I. Clinicopathological factors of unifocal and multifocal PTC.

\begin{tabular}{lccr}
\hline Clinicopathological factor & Unifocal PTC $(\mathrm{n}=312)$ & Multifocal PTC $(\mathrm{n}=35)$ & P-value \\
\hline Age, years & $48.3 \pm 2.4$ & $45.1 \pm 2.7$ & 0.86 \\
Male:female ratio & $96: 216$ & $10: 25$ & 0.79 \\
Hashimoto thyroiditis, no. (\%) & $21(6.7 \%)$ & $3(8.6 \%)$ & 0.68 \\
Extrathyroidal extension, no. $(\%)$ & $149(47.8 \%)$ & $23(65.7 \%)$ & 0.04 \\
Node metastases, no. $(\%)$ & $176(56.4 \%)$ & $27(77.1 \%)$ & 0.02 \\
Tall cell variant, no. $(\%)$ & $25(8.0 \%)$ & $2(5.7 \%)$ & 0.63 \\
T1/2:T3/4 ratio & $149: 163$ & $10: 25$ & 0.03 \\
Recurrence, no. $(\%)$ & $3(0.9 \%)$ & $5(14.3 \%)$ & 0.00
\end{tabular}

PTC, papillary thyroid carcinoma.

following conditions: i) tumor size $>1 \mathrm{~cm}$; ii) extrathyroidal extension; iii) palpable lymph nodes; and iv) bilateral carcinomas or ipsilateral disease with nodules in the contralateral lobe. Hemithyroidectomy was only performed on patients with ipsilateral disease and none of the four risk conditions. Central neck dissection (CND) was routinely performed on every patient. Lateral neck dissection was performed when the lateral nodes were positive.

All thyroid carcinomas were pathologically classified according to World Health Organization (WHO) criteria. The mean follow-up period was $4.4 \pm 0.2$ years at the end of 2011. Ultrasonographic evaluation was performed on all patients in the first month after surgery. Every six months, ultrasonography was used for disease recurrence detection. Thyroglobulin (Tg), TSH and anti-Tg antibody measurements were also performed on patients who underwent total thyroidectomy. Recurrence was diagnosed by cytological or pathological examinations after follow-up surgery. Total thyroidectomy was performed in patients with recurrence, followed by radioactive iodide $\left({ }^{131} \mathrm{I}\right)$ treatment. Lateral neck dissection was performed in patients with lymph node recurrence. Recurrences were identified in six patients who underwent hemithyroidectomy. All the recurrences were detected in newly developed nodules in the remaining thyroid tissue. Lateral lymph node recurrence was detected in two patients who underwent total thyroidectomy.

Statistical analysis. Statistical analysis was performed using SPSS software (version 13.0; SPSS Inc., Chicago, IL, USA). Chi-squared analysis was used to compare frequencies between groups. The Kaplan-Meier method was used to analyze recurrence in a time-dependent manner. $\mathrm{P}<0.05$ was considered to indicate statistically significant differences.

\section{Results}

Patient characteristics. In the present retrospective study, 347 patients (106 males/241 females) with PTC were included. The mean age at diagnosis was $46.6 \pm 2.1$ years (range, 16-74 years). Multifocal PTC occurred in 10\% of patients $(n=35)$ and $90 \%(n=312)$ had unifocal PTC. Hemithyroidectomy was performed in $41 \%$ of unifocal PTC patients $(n=128)$ who were considered to be low-risk patients at diagnosis. Of the patients with ipsilateral multifocal PTC who were considered to be low-risk patients, $20 \%(n=7)$ underwent hemithyroidectomy. Total thyroidectomy was performed for the remaining 59\% of unifocal PTC and $80 \%$ of multifocal PTC patients. Lateral neck dissection was performed in $7 \%$ of patients $(n=24)$ with positive lateral lymph nodes.

Clinicopathological parameters of unifocal and multifocal PTC. Table I shows the clinicopathological parameters of unifocal and multifocal PTC. Age, gender, T stage, histological variants, extrathyroidal extension and lymph node metastases were comparable between the two groups. There was no significant difference between unifocal and multifocal PTC with regard to age, gender, Hashimoto thyroiditis and the tall cell variant, an aggressive PTC variant. However, patients with multifocal PTC were significantly more likely to have extrathyroidal extension. Of the patients with multifocal PTC, 65.7\% exhibited extrathyroidal extension compared with $47.8 \%$ of patients with unifocal disease $(\mathrm{P}=0.04)$. Furthermore, a significant difference was identified in the rate of lymph node metastases between the two groups, with metastases occurring in $77.1 \%$ of multifocal PTC patients vs. $56.4 \%$ of unifocal PTC patients $(\mathrm{P}=0.02)$. Higher $\mathrm{T}$ stages at diagnosis were also observed in patients with multifocal PTC. Of the multifocal PTC patients, $71 \%$ were diagnosed with stage T3/4, which was significantly higher compared with the unifocal PTC patients $(52 \%$; $\mathrm{P}=0.03)$. Moreover, the tumor recurrence rate of multifocal PTC was extremely high compared with unifocal PTC. Recurrences were identified in $14 \%$ of multifocal PTC patients compared with $<1 \%$ of patents with unifocal PTC $(\mathrm{P}=0.00)$.

Recurrence in multifocal PTC. To identify which factors account for recurrence in multifocal PTC, the clinicopathological factors affecting tumor recurrence in patients with multifocal PTC were studied and are shown in Table II. There was no significant difference between patients with and without tumor recurrence with regard to age $(\mathrm{P}=0.13)$, gender $(\mathrm{P}=0.65)$, Hashimoto thyroiditis $(\mathrm{P}=0.32)$, lymph node metastases $(\mathrm{P}=0.32)$ and the tall cell variant $(\mathrm{P}=0.55)$. Notably, recurrence was most likely to occur in patients without extrathyroidal extension and with stage T1/2 tumors. Recurrences were diagnosed in $33.3 \%$ of patients without extrathyroidal 
Table II. Effect of clinicopathological factors on recurrence of multifocal PTC patients.

\begin{tabular}{|c|c|c|c|}
\hline Clinicopathological factors & No. patients & No. recurrence $(\%)$ & P-value \\
\hline \multicolumn{4}{|l|}{ Age, years } \\
\hline$<45$ & 17 & $4(23.5)$ & \\
\hline$\geq 45$ & 18 & $1(5.6)$ & 0.13 \\
\hline \multicolumn{4}{|l|}{ Gender } \\
\hline Male & 10 & $1(10.0)$ & \\
\hline Female & 25 & $4(16.0)$ & 0.65 \\
\hline \multicolumn{4}{|l|}{ Hashimoto thyroiditis } \\
\hline Yes & 3 & $1(33.3)$ & \\
\hline No & 32 & $4(12.5)$ & 0.32 \\
\hline \multicolumn{4}{|l|}{ Extrathyroidal extension } \\
\hline Yes & 23 & $1(4.4)$ & \\
\hline No & 12 & $4(33.3)$ & 0.02 \\
\hline \multicolumn{4}{|l|}{ Node metastases } \\
\hline Yes & 27 & $3(11.1)$ & \\
\hline No & 8 & $2(25.0)$ & 0.32 \\
\hline \multicolumn{4}{|l|}{ Tall cell variant } \\
\hline Yes & 2 & 0 & \\
\hline No & 33 & $5(15.2)$ & 0.55 \\
\hline \multicolumn{4}{|l|}{ T stage } \\
\hline $\mathrm{T} 1 / 2$ & 10 & $4(40.0)$ & \\
\hline $\mathrm{T} 3 / 4$ & 25 & $1(4.0)$ & 0.01 \\
\hline \multicolumn{4}{|l|}{ Surgical treatment } \\
\hline Hemithyroidectomy & 7 & $4(57.1)$ & \\
\hline Total thyroidectomy & 28 & $1(3.6)$ & 0.00 \\
\hline
\end{tabular}

PTC, papillary thyroid carcinoma.

extension and $40 \%$ of $\mathrm{T} 1 / 2$ patients within the 5 -year follow-up period $(\mathrm{P}=0.02, \mathrm{P}=0.01$, respectively). These patients were considered to be low-risk. Hemithyroidectomy was performed if the patients had ipsilateral disease at diagnosis and none of the following conditions: i) extrathyroidal extension; ii) palpable lymph nodes; and iii) bilateral carcinomas or ipsilateral disease with nodules on the contralateral lobe. Surgical treatment was supposed to be involved with disease recurrence in this regard. In the present study, there were seven ipsilateral multifocal PTC patients who were defined as low risk and underwent hemithyroidectomy. Recurrences were identified in $57 \%$ of patients undergoing hemithyroidectomy compared with $3.6 \%$ of patents who underwent total thyroidectomy $(\mathrm{P}=0.00)$. This showed that hemithyroidectomy resulted in more frequent recurrence in multifocal PTC patients. This result was consistent with the observation that recurrences were most likely to be identified in low-risk multifocal PTC patients, since hemithyroidectomy was only performed in the low-risk group.

Involvement of hemithyroidectomy in recurrence among unifocal PTC patients. To identify whether hemithyroidectomy also resulted in more frequent cases of recurrence in unifocal PTC patients, the number of patients with recur- rent PTC was obtained and the patients were grouped by hemithyroidectomy and total thyroidectomy (Table III). All recurrences were located in the remaining thyroid tissue in the patients who underwent hemithyroidectomy. Recurrence of the lateral lymph nodes was detected in 2 patients who underwent total thyroidectomy. The recurrence rate of the multifocal PTC patients who underwent hemithyroidectomy was significantly higher than that of the unifocal PTC patients (57.1 vs. 1.6\%, $\mathrm{P}=0.00)$. However, there was no significant difference between the multifocal and unifocal PTC groups who underwent total thyroidectomy (3.6 vs. $0.5 \%, \mathrm{P}=0.12$ ).

Comparison of multifocal PTC patients undergoing hemithyroidectomy and total thyroidectomy. To further understand the difference in the recurrence rate between multifocal PTC patients undergoing hemithyroidectomy and patients undergoing total thyroidectomy, recurrence was analyzed in a time-dependant manner. Recurrences were identified in four out of seven multifocal PTC patients who underwent hemithyroidectomy. The median recurrence time was $40.0 \pm 6.55$ months. Only one case of recurrence was identified in the patients who underwent total thyroidectomy. The recurrence rate of the multifocal PTC patients who underwent hemithyroidectomy was significantly higher than that of 
Table III. Effect of surgical procedures on recurrence of unifocal and multifocal PTC patients.

\begin{tabular}{lccr}
\hline & No. unifocal PTC $(\%)$ & No. multifocal PTC (\%) & P-value \\
\hline $\begin{array}{l}\text { Recurrence after hemithyroidectomy } \\
\text { Yes }\end{array}$ & $2(1.6)$ & $4(57.1)$ & 0.00 \\
No & $126(98.4)$ & $3(42.9)$ & $1(3.6)$ \\
Recurrence after total thyroidectomy & $1(0.5)$ & $27(96.4)$ & 0.12 \\
Yes & $183(99.5)$ & & \\
No & & & \\
\hline PTC, papillary thyroid carcinoma. &
\end{tabular}

Table IV. Analysis of recurrence in a time-dependant manner according to hemithyroidectomy and total thyroidectomy.

\begin{tabular}{lcccc}
\hline & No. patients & No. recurrences & Median recurrence time (months) & P-value \\
\hline Hemithyroidectomy & 7 & 4 & $40.0 \pm 6.55$ & 0.00 \\
Total thyroidectomy & 28 & 1 & - & \\
\hline
\end{tabular}

the patients who underwent total thyroidectomy $(\mathrm{P}=0.00$; Table IV).

\section{Discussion}

PTC is characterized by increased multifocal disease, frequent clinical nodal metastases (20-50\%) and an even higher rate of subclinical micrometastases (50-90\%) (4-9). PTC is often clinically multifocal carcinoma that presents within a single thyroid gland or pluriform. The frequency of multifocal PTC is $30-40 \%$ (1-3). The frequency of multifocality in the present study was $10 \%$, which is relatively low. The pathogenesis of multifocality remains unknown. Several factors, such as iodine intake and single nucleotide polymorphisms (SNPs), may be involved in the multicentricity of PTC. However, further research into the origin of multicentricity is required.

There are two unresolved issues in the treatment of multifocal PTC: the necessity of elective CND as opposed to therapeutic CND (15) and the necessity of total thyroidectomy as opposed to hemithyroidectomy $(10,16)$, particularly in developing countries. The debate about how to treat clinically-negative lymph nodes has arisen as lymph node metastases are not considered to be prognostic factors for poor survival in well-differentiated PTC patients (17). However, certain studies have suggested that a high proportion (80-90\%) of patients exhibit subclinical lymph node metastases (micrometastases) at the time of surgical intervention (6-9). Furthermore, multifocal thyroid disease has been associated with distant metastases in certain studies $(18,19)$, suggesting that multifocal PTC carries an increased risk of metastases.

In the present study, the rate of lymph nodes metastasis was compared between unifocal and multifocal PTC. A significantly higher proportion of patients with lymph node metastases were identified in the multifocal PTC group, indicating that the risk of metastasis is correlated with the multifocality of the primary tumor. Since regional metastases in patients older than 45 years do indicate significantly negative prognoses (20), elective CND is likely to be most beneficial for multifocal PTC patients, particularly those classified as high risk, since follow-up surgery is considered to be difficult in the central compartment in cases of disease recurrence $(21,22)$.

The necessity of total thyroidectomy as opposed to hemithyroidectomy has been another widely debated issue for several decades (23). Although the American Thyroid Association (ATA) has suggested that all patients with multifocal PTC should undergo total thyroidectomy, hemithyroidectomy is often performed on patients with multifocal PTC to reduce complications. This argument is based on the problem that these carcinomas are either intrathyroidal metastases from the primary tumor spreading through the lymphatic system or independent carcinomas. Activating mutations in BRAF are particularly common in adults with PTC. In a number of studies, the presence of BRAF mutations was associated with more aggressive clinical courses (24-26). Thus, analysis of the distribution of BRAF aids in understanding the origins of multifocal carcinomas.

A number of studies have suggested that multifocal carcinomas have independent origins due to the heterogeneous distribution of BRAF mutations in multifocal PTC (10-13). These findings indicate that any thyroid tissue remaining in patients with multifocal PTC after surgery may contain, or be likely to develop, additional cancer foci which may become recurrences (10). This is consistent with the present observation that the recurrence rate of the multifocal PTC patients who underwent hemithyroidectomy was significantly higher than that of the unifocal PTC patients (57.1 vs. $1.6 \%, \mathrm{P}=0.00$ ). Notably, according to the present data it was the low-risk multifocal PTC patients, not the high-risk patients, who had a higher recurrence rate. These patients were expected to be less vulnerable to disease recurrence and poor prognosis. The data in Tables III and IV suggest that this is due to the fact that only the low-risk patients in this study underwent 
hemithyroidectomy instead of total thyroidectomy. It also appears that the high-risk patients were able to avoid disease recurrence as total thyroidectomy was performed in this group of patients. The observation that only $1.6 \%$ of patients with unifocal PTC who underwent hemithyroidectomy developed recurrence supports the theory that multifocal carcinomas have independent origins. The development of new cancer foci in the remaining thyroid tissue leads to disease recurrence in multifocal PTC patients undergoing hemithyroidectomy.

Thus, the decision on whether to perform hemithyroidectomy for patients with ipsilateral multifocal PTC should only be made after careful deliberation. However, it is clear that certain papillary cancer foci may have the same origin since there are mutlifocal carcinomas with homogeneous distributions of BRAF mutations or lacking BRAF mutations (27). A randomized clinical trial may resolve this issue with more certainty.

\section{Acknowledgements}

The authors would like to thank Dr Krishnan Rangaswamy Iyengar (James Cook University, Queensland, Australia) for critically reading and discussing the manuscript.

\section{References}

1. Sherman SI: Thyroid carcinoma. Lancet 361: 501-511, 2003.

2. Hundahl SA, Fleming ID, Fremgen AM and Menck HR: A National Cancer Data Base report on 53,856 cases of thyroid carcinoma treated in the U.S., 1985-1995. Cancer 83: 2638-2648, 1998.

3. Caron NR and Clark OH: Well differentiated thyroid cancer. Scand J Surg 93: 261-271, 2004.

4. Scheumann GF, Gimm O, Wegener G, Hundeshagen H and Dralle H: Prognostic significance and surgical management of locoregional lymph node metastases in papillary thyroid cancer. World J Surg 18: 559-567, 1994.

5. Wang TS, Dubner S, Sznyter LA and Heller KS: Incidence of metastatic well-differentiated thyroid cancer in cervical lymph nodes. Arch Otolaryngol Head Neck Surg 130: 110-113, 2004.

6. Chow SM, Law SC, Chan JK, Au SK, Yau S and Lau WH: Papillary microcarcinoma of the thyroid: prognostic significance of lymph node metastasis and multifocality. Cancer 98: 31-40, 2003.

7. Qubain SW, Nakano S, Baba M, Takao S and Aikou T: Distribution of lymph node micrometastasis in pNO well-differentiated thyroid carcinoma. Surgery 131: 249-256, 2002.

8. Arturi F, Russo D, Giuffrida D, et al: Early diagnosis by genetic analysis of differentiated thyroid cancer metastases in small lymph nodes. J Clin Endocrinol Metab 82: 1638-1641, 1997.

9. Wada N, Duh QY, Sugino K, et al: Lymph node metastasis from 259 papillary thyroid microcarcinomas: frequency, pattern of occurrence and recurrence, and optimal strategy for neck dissection. Ann Surg 237: 399-407, 2003.

10. Shattuck TM, Westra WH, Ladenson PW and Arnold A: Independent clonal origins of distinct tumor foci in multifocal papillary thyroid carcinoma. N Engl J Med 352: 2406-2412, 2005.
11. Morris LG and Myssiorek D: Improved detection does not fully explain the rising incidence of well-differentiated thyroid cancer: a population-based analysis. Am J Surg 200: 454-461, 2010.

12. Giannini R, Ugolini C, Lupi C, et al: The heterogeneous distribution of BRAF mutation supports the independent clonal origin of distinct tumor foci in multifocal papillary thyroid carcinoma. J Clin Endocrinol Metab 92: 3511-3516, 2007.

13. Park SY, Park YJ, Lee YJ, et al: Analysis of differential BRAF(V600E) mutational status in multifocal papillary thyroid carcinoma: evidence of independent clonal origin in distinct tumor foci. Cancer 107: 1831-1838, 2006.

14. Hay ID, Thompson GB, Grant CS, et al: Papillary thyroid carcinoma managed at the Mayo Clinic during six decades (1940-1999): temporal trends in initial therapy and long-term outcome in 2444 consecutively treated patients. World J Surg 26: 879-885, 2002

15. Salter KD, Andersen PE, Cohen JI, et al: Central nodal metastases in papillary thyroid carcinoma based on tumor histologic type and focality. Arch Otolaryngol Head Neck Surg 136: 692-696, 2010.

16. Mazeh H, Samet Y, Hochstein D, et al: Multifocality in well-differentiated thyroid carcinomas calls for total thyroidectomy. Am J Surg 201: 770-775, 2011.

17. Shaha AR: Implications of prognostic factors and risk groups in the management of differentiated thyroid cancer. Laryngoscope 114: 393-402, 2004.

18. Katoh R, Sasaki J, Kurihara H, Suzuki K, Iida Y and Kawaoi A: Multiple thyroid involvement (intraglandular metastasis) in papillary thyroid carcinoma: a clinicopathologic study of 105 consecutive patients. Cancer 70: 1585-1590, 1992.

19. Tscholl-Ducommun J and Hedinger CE: Papillary thyroid carcinomas: morphology and prognosis. Virchows Arch A Pathol Anat Histol 396: 19-39, 1982.

20. Hughes CJ, Shaha AR, Shah JP and Loree TR: Impact of lymph node metastases in differentiated carcinoma of the thyroid: a matched-pair analysis. Head Neck 18: 127-132, 1996.

21. White ML, Gauger PG and Doherty GM: Central lymph node dissection in differentiated thyroid cancer. World J Surg 31: 895-904, 2007.

22. Shindo M, Wu JC, Park EE and Tanzella F: The importance of central compartment elective lymph node excision in the staging and treatment of papillary thyroid cancer. Arch Otolaryngol Head Neck Surg 132: 650-654, 2006.

23. Mazzaferri EL and Kloos RT: Clinical review 128: Current approaches to primary therapy for papillary and follicular thyroid cancer. J Clin Endocrinol Metab 86: 1447-1463, 2001

24. Xing M: BRAF mutation in thyroid cancer. Endocr Relat Cancer 12: 245-262, 2005.

25. Riesco-Eizaguirre G, Gutiérrez-Martínez P, García-Cabezas MA, Nistal M and Santisteban P: The oncogene BRAF V600E is associated with a high risk of recurrence and less differentiated papillary thyroid carcinoma due to the impairment of $\mathrm{Na}^{+} / \mathrm{I}$ targeting to the membrane. Endocr Relat Cancer 13: 257-269, 2006.

26. Lee X, Gao M, Ji Y, Yu Y, Feng Y, Li Y, et al: Analysis of differential BRAF(V600E) mutational status in high aggressive papillary thyroid microcarcinoma. Ann Surg Oncol 16: 240-245, 2009.

27. Jovanovic L, Delahunt B, McIver B, Eberhardt NL and Grebe SK: Most multifocal papillary thyroid carcinomas acquire genetic and morphotype diversity through subclonal evolution following the intra-glandular spread of the initial neoplastic clone. J Pathol 215: $1451-1454,2008$. 